\title{
Synergistic behavioral responses of female oriental fruit moths (Lepidoptera:Tortricidae) to synthetic host plant-derived mixtures are mirrored by odor-evoked calcium activity in their antennal lobes
}

\author{
Jaime Ci Piñero ${ }^{\mathrm{a}, \mathrm{l}}$, C. Giovanni Galizia ${ }^{\mathrm{b}}$, Silvia Dorn ${ }^{\mathrm{a}, *}$ \\ ${ }^{a}$ ETH Zurich, Institute of Plant Sciences/Applied Entomology, Schmelzbergstrasse 9/LFO, 8092 Zurich, Switzerland \\ bepartment of Biology, University of Konstanz, D78457 Konstanz, Germany
}

\begin{abstract}
Attraction of many gravid female herbivore insects to suitable host plants is mediated largely by olfactory cues. Behaviorally, synergism among odor mixtures constituents underlies this attraction in some systems. Yet, the representation of synergistic odor-mixture effects is unknown in the antennal lobe, the first processing center for olfactory information in insect brains. Using both behavioral and physiological data we demonstrate that in the oriental fruit moth, Cydia (Grapholita) molesta, a minor constituent of a plant-derived synthetic mixture plays a key role in behavioral discrimination and in neural representation of mixtures. Behaviorally, minute amounts of benzonitrile added to an unattractive 4-compound mixture resulted in a bioactive 5-compound mixture that was as attractive to mated female moths as the natural blend. Physiologically, the bioactive benzonitrile-containing mixture elicited strong activation of one additional, new type of glomerulus that showed specific synergisms for this mixture. The specific pattern of activated glomeruli elicited by the addition of benzonitrile demonstrates a physiological correlate to the behaviorally observed synergism, and emphasizes the key role of a minor component of a complex mixture. While minor constituents of mixtures are often overlooked, they may, as conclusively documented here, be determinant for successful recognition and behavioral discrimination of suitable host plants by herbivore insects.
\end{abstract}

Keywords: Behavior; Calcium imaging; Antennal lobe; Olfactory glomeruli; Odor mixture; Synergism; Benzonitrile

\section{Introduction}

Most animals rely on olfaction as the main chemosensory modality to successfully distinguish and discriminate chemical cues that are essential for their reproduction and survival (Lledo et al., 2005). The olfactory system of herbivore insects has long been recognized to possess an outstanding ability to filter out odors that are behaviorally meaningful from the complex volatile blends that are commonly emitted by both host and non-host plants (Schoonhoven et al., 1998). In addition to blend complexity, seasonal and daily variations in abundance and relative

\footnotetext{
${ }^{*}$ Corresponding author. Tel.: +41446323921 ; fax: +41446321171.

E-mail address: silvia.dorn@ipw.agrl.ethz.ch (S. Dorn).

'Present address. College of Tropical Agriculture and Human Resources, University of Hawaii at Manoa, 3050 Maile Way, Honolulu, HI 96822, USA.
}

ratios of compounds (Vallat and Dorn, 2005; Vallat et al., 2005) may further complicate the successful detection of a suitable host plant by an individual herbivore searching for resources. How can the olfactory system of herbivore insects recognize and discriminate chemical signals that convey essential information about the identity and suitability of host plants considering the low signal-tonoise ratio of relevant mixtures in their natural habitats?

At the behavioral level, mixtures of host plant-derived compounds rather than single constituents are required in many systems to elicit appropriate levels of response of herbivore insects (Carlsson and Hansson, 2003; Bruce, 2005). Furthermore, synergistic interactions among the components of an odor blend have been proposed to contribute to the attraction of some species of insects to their host plants (Visser, 1986). Empirical evidence for synergism between general green leaf volatiles and specific aromatic compounds 
in the attraction of gravid female moths was documented recently in the oriental fruit moth, Cydia (Grapholita) molesta (Busck) (Lepidoptera:Tortricidae) (Piñero and Dorn, 2007). A combination of three green leaf volatiles with benzonitrile and benzaldehyde was attractive, while neither benzaldehyde, benzonitrile nor the blend of the three green leaf volalites were attractive to the female moths when tested separately. The synthetic 5-compound mixture identified by Pinero and Dorn (2007) is nature-identical as it reflects relative ratios of constituents (Natale et al., 2003) present in the headspace of peach shoots (Prunus persica [L.] Batsch; Rosaceae), the main host plant of this moth. In another tortricid moth, Lobesia botrana (Den. and Schiff.), three ubiquitous compounds were found to act synergistically in attracting mated females (Tasin et al., 2007).

From a sensory processing perspective, no published study has yet elucidated how the volatile chemical information contained in plant-derived odor mixtures that elicit synergistic behavioral responses of herbivore insects is encoded and subsequently integrated into behaviorally relevant brain representations. While mixture interactions, and in particular synergism, are well known in the pheromone system (e.g., Christensen et al., 1991; Mayer and Doolittle, 1995; Nikonov and Leal, 2002), to our knowledge no information is available on synergistic interaction of host plant volatiles in the antennal lobes (AL) of herbivore insects. Such information is essential for a better understanding of olfactory sensory coding as well as of mechanisms underlying host preference (Smith and Getz, 1994). In this study we combined behavioral data with calcium imaging to gain insight into the mechanisms underlying behavioral and neuronal responses to odor mixtures in the oriental fruit moth. This herbivore insect reproduces predominantly in plants belonging to the family Rosaceae (Rothschild and Vickers, 1991), and mated females are strongly attracted to the natural blend of peach-shoot volatiles (Natale et al., 2003). In particular, we aimed at (1) behaviorally, quantifying the olfactory response of female $C$. molesta to different synthetic mixtures and to the full blend of volatiles emitted by peach shoots in dual choice bioassays; (2) physiologically, characterizing the pattern of glomeruli activated by three odor mixtures and their individual constituents in the $\mathrm{AL}$ of the female moths as well as the dose-response relationships of each odorant to determine potential mixture interactions; and (3) for the mixture-sensitive glomerulus that showed the strongest responses to the synergistic mixture, measuring calcium responses to different mixtures that varied in the number and type of constituents when compared with signals educed by benzonitrile alone.

\section{Materials and methods}

\subsection{Insects}

The laboratory strain of $C$. molesta, originating from BioTechnologie B.T. (Todi-Perigua, Italy), was reared on artificial diet for over 150 generations (Hughes et al., 2004). Upon emergence, test females were held in containers in groups of ca. 20 females together with ca. 30 males to ensure mating, at $26^{\circ} \mathrm{C}, 70 \%$ relative humidity (r.h.) and $16 \mathrm{~h}$ light: $8 \mathrm{~h}$ darkness. All females tested were $2-3$ days old, presumably mated, and deprived of food but with ad libitum access to water. They had not been exposed to natural or synthetic plant volatiles prior to the experiments.

\subsection{Chemicals}

Compounds used for the experiments were the green leaf volatiles (Z)-3-hexen-1-ol (Sigma-Aldrich Inc., St. Louis MO, USA, purity >99\%), (E)-2-hexenal (Fluka, Buchs, Switzerland, purity $>99 \%$ ), and $(Z)-3$-hexen-1-yl acetate (Sigma-Aldrich, purity $>99 \%$ ), and the aromatics benzaldehyde (Fluka, Buchs, Switzerland, purity $>99 \%$ ) and benzonitrile (Fluka, purity $>99 \%$ ). In addition, the monoterpenoid $( \pm$ ) linalool (Fluka, purity $>95 \%$ ) was evaluated in the physiological experiments as an external standard to normalize the magnitude of calcium responses between test insects.

\subsection{Behavioral bioassays}

We investigated the short-range olfactory response of female moths to different synthetic mixtures prepared using two different types of solvents, and compared the attractiveness of a bioactive mixture versus natural peach shoot volatiles in a dual choice bioassay consisting of an all-glass Y-tube olfactometer. This approach allows for detailed analysis of short-range olfactory responses of insects (Eigenbrode and Bernays, 1997). In short, the olfactometer setup consisted of a Y-tube (arm length: $20 \mathrm{~cm}$ each) connected to one of two chambers (length: $35 \mathrm{~cm}$, diameter: $6 \mathrm{~cm}$ ) containing a particular test odor or a control odor. Moistened, activated charcoal-filtered air was pumped into each of two chambers at a rate of $740 \pm 10 \mathrm{~mL} / \mathrm{min}$ at the entrance. A silicon/teflon septum (diameter: $13 \mathrm{~mm}$ ) (Supelco, Bellefonte, PA, USA) was loaded with an amount (specified below) of a particular test mixture or the solvent (control) and placed inside one of the two chambers. In one case, the control chamber was loaded with a peach twig as detailed below. The behavioral response of individual females, released at the entrance of the common arm of the Y-tube, was recorded invariably during a 10-min period (Vallat and Dorn, 2005). The outcome was classified as no-choice if the test female remained in the common arm and as choice if the female entered either the test arm having a particular odor mixture or the control arm (Piñero and Dorn, 2007). In the rare event a test female made multiple choices, only the first choice was considered for the analyses. Bioassays were conducted exclusively during the $2.5 \mathrm{~h}$ preceding the scotophase (Natale et al., 2003) at $24-26^{\circ} \mathrm{C}$ and $60-70 \%$ 
r.h. A $60 \mathrm{~W}$ red bulb allowed for behavior observation (Hern and Dorn, 2004).

The composition of each the three mixtures evaluated was: 3 -compound mixture: $(Z)-3$-hexen-1-yl acetate, $(Z)-3$ hexen-1-ol, and benzaldehyde (ratio: 71.45:14.98:13.57); 4-compound mixture: the same three compounds present in the first mixture plus $(E)$-2-hexenal (ratio: $69.84: 14.64$ : 13.26:2.26); 5-compound mixture: the four aforementioned compounds plus benzonitrile (ratio: 69.74:14.62:13.24: 2.25:0.15). Ratios (shown in parenthesis) correspond to the natural ratios of compounds present in the headspace of peach shoots, known to comprise over 20 compounds (Natale et al, 2003).

The specific pair-wise comparisons of odors were: (a) using $n$-hexane as solvent, each of three synthetic mixtures comprising either three, four or five compounds $(50 \mu \mathrm{L}$ in each case) versus $50 \mu \mathrm{L}$ of $n$-hexane (control), and (b) using mineral oil as solvent, each of two mixtures comprising either four or five compounds $\left(1 \mu \mathrm{L}\right.$ of mixture at $\left.10^{-4}\right)$ against $1 \mu \mathrm{L}$ of the solvent (control); in a subsequent test we compared the bioactive 5-compound mixture $(5 \mu \mathrm{L}$ of mixture at $10^{-4}$ ) versus the natural blend of volatiles emitted by peach shoots. Peach shoots of $\sim 3.0 \mathrm{~cm}$ in length and containing 3-4 leaves were excised from potted 1-yearold peach trees (cultivar Alexander) ca. $15 \mathrm{~min}$ before the start of bioassays, wrapped at the cut end with Parafilm (Pechiney Plastic Packaging, Chicago, IL), and introduced into the control chamber of the $Y$-tube.

For the set of experiments that involved $n$-hexane (Fluka, purity $>99 \%$ ) as solvent, mixtures were prepared at $46 \mathrm{ppm}$ for the most abundant compound based on Piñero and Dorn (2007). For the set of comparisons that involved mineral oil as solvent, the two mixtures evaluated comprising either four or five compounds were prepared in the same way as for the physiological experiments (described below).

For each of the six pair-wise comparisons listed above, the sample size consisted of 65 females except for the comparison of the 5-compound mixture prepared in mineral oil versus peach shoot volatiles $(n=50)$. A total of 375 individual female moths were tested in the behavioral bioassays.

\subsection{Physiological experiment 1 : calcium signals to synthetic mixtures and individual components}

This experiment aimed at investigating glomerular activity patterns in the AL of mated female moths evoked by each of the mixtures evaluated at the behavioral level, as well as their individual constituents, using the bath-applied calcium-sensitive dye technique. This approach measures in real time and in vivo odor-evoked glomerular activity patterns by quantifying changes in intracellular calcium concentrations (Galizia and Menzel, 2001), and offers a very good spatial resolution with good signal-to-noise ratio (Galizia and Vetter, 2005). It has been used with high success to study coding of individual odors and odor mixtures in the brain of honeybees (e.g., Galizia et al, 1998; Sachse et al., 1999), as well as in the moths Heliothis virescens (Fabricius) (Galizia et al., 2000; Skiri et al., 2004) and Spodoptera littoralis (Boisduval) (Carlsson et al., 2007).

Each of the individual odors and odor mixtures tested were prepared and kept in $20-\mathrm{mL}$ glass vials filled with nitrogen (in the gas phase, to avoid oxidation) before cramping. Each odorant was tested at three different concentrations $\left(10^{-2}, 10^{-3}\right.$ and $\left.10^{-4}\right)$ using odorless mineral oil (Aldrich spectroscopy) as solvent. For the preparation of the mixtures, the amount of $(Z)-3$-hexen-1-yl acetate was kept constant and the ratios of the remaining compounds were adjusted accordingly. Stimulations of the antennae with pure mineral oil (solvent), with $( \pm)$ linalool (at $10^{-2}$ ), and with the carrier nitrogen (thereafter referred to as "blank") served as controls.

For optical recordings, individual female moths were mounted on a custom-made Plexiglass stage, fixed with wax and dissected using the technique described by Galizia and Vetter (2005). Briefly, after exposing the AL, they were stained for $50 \mathrm{~min}$ by bath-applying $10 \mu \mathrm{L}$ of the dye Calcium Green 2 AM (Molecular Probes, Eugene, OR, dissolved in saline with Pluronic and DMSO) to the open brain cavity. The brain was kept in saline (in $\mathrm{mM}$ : 130 $\mathrm{NaCl}, 6 \mathrm{KCl}, 4 \mathrm{MgCl}_{2}, 5 \mathrm{CaCl}_{2}, 160$ sucrose, $25 \mathrm{D}$-glucose, 10 HEPES free acid, pH 6.7, 500 mOsmol; all chemicals from Sigma-Aldrich, Germany) at all times. After removing excess dye, the preparation was placed under an upright microscope with a $20 \times$ water-immersion physiology objective $(\mathrm{NA}=0.9)$. A constant stream of clean air $(60 \mathrm{~mL} / \mathrm{s})$ was blown over the antennae, which was replaced by odor-loaded air during odor delivery. The entire preparation was kept in a wind tunnel of charcoalfiltered and temperature controlled air (speed: $1.3 \mathrm{~m} / \mathrm{s}$, $21-23^{\circ} \mathrm{C}$ ) to avoid olfactory contamination from environmental odors in the room.

Stimuli were applied in succession, always starting with the lowest concentration, using a motor-driven computerized system (Combi PAL; CTC Analytics AG, Switzerland) as double $1 \mathrm{~s}$ pulses, at 3 and $6 \mathrm{~s}, 1 \mathrm{~mL} / \mathrm{s}$ each. Physiological responses to blank, to $\left( \pm\right.$ ) linalool $\left(\right.$ at $10^{-2}$ ) and to the solvent mineral oil were recorded at least three times (start, mid and end) during an experiment. In general, measurements on a single animal lasted about $1.5 \mathrm{~h}$. For each animal tested, the order of odors (i.e., block of concentration series) was randomly assigned in order to minimize effect of odor position.

During optical recording, 52 frames (images) were taken for each stimulus at a frequency of $4 \mathrm{~Hz}$ (i.e., 4 frames per second) with $240 \mathrm{~ms}$ exposure time per image. Recordings were made using a CCD camera (Imago QE, Photonics) mounted to the microscope. An $8 \times 8$ binning on chip was applied to an optical resolution of $4.6 \mu \mathrm{m} \times 4.6 \mu \mathrm{m}$ per pixel. Excitation light was $475 \mathrm{~nm}$, emission was LP505.

Physiological responses are expressed as relative changes in fluorescence $(\Delta F / F)$, calculated from the raw data with 
the average fluorescence of frames $8-12$ as $F$. Data were filtered using spatial median filters with a size of 3 pixels to reduce noise originating from individual and isolated unproportionally bright or dark pixels and corrected for bleaching (i.e., an overall decay in fluorescence) (Galizia and Vetter, 2005). For quantitative analyses, the $\Delta F / F$ data were normalized for each moth by setting to 1 the response of the glomerulus showing the strongest response to $( \pm)$ linalool at $10^{-2}(=$ glomerulus $\mathrm{E}$; see identification of glomeruli below). This will be referred to as the reference glomerulus. Odor-evoked glomerular activity patterns are presented in false color coding (see scale in Fig. 2b). For the calculation of false-color images (depicted in Figs. 2b and c) the average value of signals recorded within $1 \mathrm{~s}$ before stimulus delivery was subtracted from the maximum value recorded within $8 \mathrm{~s}$ after stimulus delivery. This was done, for each of the glomeruli identified, within squares of $3 \times 3$ pixels corresponding to $13.8 \mu \mathrm{m}$ side length and always well within the glomerulus chosen. Time traces shown in Fig. 2d were calculated as above with the courses plotted against time. The time course of relative fluorescence changes shown in Figs. 3 and 4 was calculated for each glomerulus by averaging squares of $5 \times 5$ pixels $(23 \mu \mathrm{m} \times 23 \mu \mathrm{m})$. Analyses were conducted using custommade programs in IDL (Research Systems, Inc., now ITT Visual Information Solutions, Boulder, CO, USA).

\subsection{Physiological experiment 2: odor-evoked activity in the mixture-sensitive glomerulus}

The first physiological experiment revealed stronger calcium signals in glomerulus $\mathrm{A}$ when benzonitrile was included in the 5-compound mixture at $10^{-2}$ than when benzonitrile was offered alone at $10^{-4}$ (Fig. 2). This result is remarkable because the amount of benzonitrile alone at $10^{-4}(\sim 100.4 \mu \mathrm{L})$ is about 4.5 times greater than the amount of this compound present in the bioactive mixture at $10^{-2}(\sim 22.1 \mu \mathrm{L})$. The second physiological experiment (Fig. 5) was aimed at further elaborating on this synergistic interaction. Calcium signals were recorded in glomerulus $\mathrm{A}$ in response to stimulation by each of the following odorants: (1) the bioactive 5-compound mixture; (2) the 4-compound mixture that excluded benzonitrile, tested in behavior and in the first physiological experiment; (3) a 4compound mixture that included benzonitrile but excluded benzaldehyde; (4) a binary mixture consisting of benzaldehyde plus benzonitrile; and (5) benzonitrile alone.

Because the objective of this experiment was to determine the relative contribution of some constituents of the mixtures in eliciting calcium responses in glomerulus A when compared to benzonitrile alone, it was critical that the concentration of each of the components of the mixtures was kept constant. This was achieved by substituting those compounds missing from the 5-compound mixture (listed above) by an equivalent amount of mineral oil. After preparation of each mixture at the highest concentration tested (i.e., $10^{-2}$ ), we used serial dilutions with mineral oil to generate three additional concentrations $\left(10^{-3}-10^{-5}\right)$.

Preparation of the female moths, odor delivery and optical recordings was as described above. Moths in the second physiological experiment may have possessed a broader genetic diversity than in the first experiment since the laboratory colony of $C$. molesta had been refreshed by crossing-in wild adults that originated from field-collected infested fruit in Italy.

\subsection{Identification of glomeruli}

While we could not identify glomeruli on the basis of their morphology, it was possible to recognize homologous glomeruli across individuals on the basis of their physiological responses to the different odorants, allowing for inter-individual statistical analysis. Six different glomeruli were identified based on their characteristic strong responses to the individual odorants evaluated at the lowest concentration $\left(10^{-4}\right)$ in the first physiological experiment. Glomerulus A: strongest response to benzonitrile at low concentrations (responses of this glomerulus to benzonitrile were present at odor concentrations as low as $10^{-7}$, data not shown). In some of the moths tested this glomerulus saturated its response to benzonitrile at concentration $10^{-2}$ (see Fig. 4), and responded to benzaldehyde instead, a characteristic indicative of its complex response properties, some of which were investigated here. Glomerulus A showed to be highly sensitive to the 5-compound mixture that contained benzonitrile. Glomerulus B showed the strongest response to benzaldehyde, whereas glomerulus $\mathrm{C}$ exhibited strong responses to the three green leaf volatiles tested and to $( \pm)$ linalool (at $10^{-2}$ ). Glomerulus $\mathrm{D}$ was the second glomerulus showing strong responses to green leaf volatiles as well as to ( \pm ) linalool $\left(\right.$ at $10^{-2}$ ). For glomerulus $E$ the strongest response was to the standard $( \pm)$ linalool $\left(10^{-2}\right)$ and therefore this glomerulus was considered the reference glomerulus, used to normalize responses across individual females (as described above). Glomerulus $F$ frequently showed the second strongest response to $( \pm)$ linalool $\left(10^{-2}\right)$.

\subsection{Data analysis}

Behavioral bioassays were analyzed for preference (percentage of females that made a choice, i.e., selecting either an odor mixture or the control), and for responsiveness (proportion of females that did/did not make a choice) (Bertschy et al., 1997). To test the null hypothesis of no preference, each of the comparisons described in the behavioral bioassays was analyzed using one-sample chisquare tests on the numbers of female moths that made a choice. Pair-wise comparisons were made to test for possible differences between mixtures on level of female responsiveness. Comparisons were made separately for bioassays that used the same solvent. The $t$-value for the respective comparison was used as a basis to calculate the $P$ 
level (StatSoft, 2001). For the three pair-wise comparisons that are based on the solvent $n$-hexane, Bonferroni (1936) correction was used; here the alpha value was lowered to 0.017 . Female responsiveness was compared directly for the 4. and 5-compound mixtures for the tests that used mineral oil as solvent.

Data from the two physiological experiments were analyzed using one-way ANOVA to compare, across females, normalized calcium signals in response to the different mixtures evaluated. For the first physiological experiment a $t$-test was also used to contrast, across females, normalized calcium signals in the benzonitrile glomerulus elicited by benzonitrile alone (at $10^{-4}$ ) versus signals elicited by the 5-compound mixture (at $10^{-2}$ ). Tukey HSD tests were used whenever appropriate to separate treatment means. Statistical analyses were conducted using Statistica (StatSoft, 2001).

\section{Results}

\subsection{Behavioral bioassays}

Odor mixtures comprising 3 or 4 compounds did not elicit a significant preference in female oriental fruit moths when tested against the solvent $n$-hexane in the olfactory bioassay (Fig. 1a). Benzonitrile alone when tested against $n$-hexane as the solvent control was previously found to be behaviorally ineffective (50\% choice for odor, $50 \%$ for control, $P=1.0$ ) (Piñero and Dorn 2007). However, addition of trace amounts of benzonitrile $(0.15 \%$ of the mixture) to the 4-compound mixture elicited a significant preference for the resulting 5-compound mixture. The percentage of females that did not make a choice was significantly lower for the 5-compound mixture than for the 3-compound mixture $(P<0.017$ after Bonferroni correction) but not than for the 4-compound mixture $(P>0.017$ after Bonferroni correction) (Fig. 1a).

To exclude any potential behavioral effect of $n$-hexane, this solvent was replaced by mineral oil in a successive test. No preference was recorded when females were given a choice between the 4-compound mixture and mineral oil. In contrast, the benzonitrile-containing 5-compound mixture elicited a significant preference of the female moths (Fig. 1b). The attractant effect of the 5-compound mixture was thus confirmed, indicating that the solvent hexane did not play a significant role in the preference of the female moths. Remarkably, the preference of the female moths for the 5-compound mixture was not significantly different from the full blend of volatiles emitted by shoots of the main host plant, peach (Fig. 1b). The percentage of females not making a choice did not differ significantly between the 4- and the 5-compound mixtures $(P>0.05)$.

\subsection{Calcium signals to synthetic mixtures and individual components}

A morphological view of the AL of one female moth is shown in Fig. 2a. Each of the three odor mixtures evaluated (two are shown in Fig. $2 b$ for the same individual) induced strong calcium signals in the $A L$. These signals were spatially structured and corresponded to patterns consisting of individual glomeruli. In general, the pattern of response of the different glomeruli activated

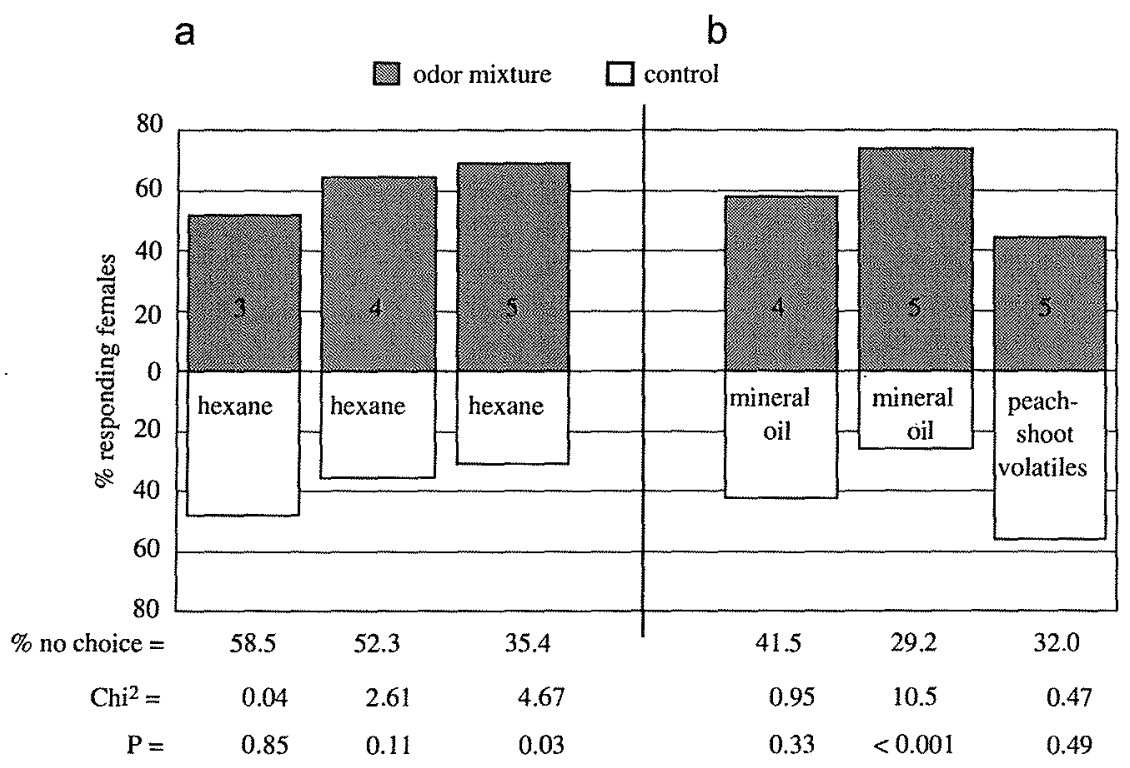

Fig. 1. Behavioral responses of mated female oriental fruit moth to synthetic mixtures depend on synergistic interactions among odor constituents: (a) response to each of three synthetic mixtures comprising either 3,4 , or 5 compounds versus the solvent $n$-hexane; (b) response to either a 4 - or a 5 compound mixture prepared in mineral oil versus this solvent, and response to the 5 -compound mixture (prepared in mineral oil) versus peach shoot volatiles. 
a

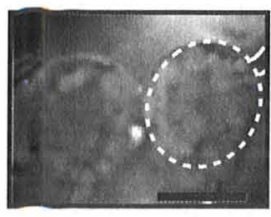

c

(i)

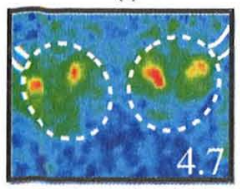

(iv)

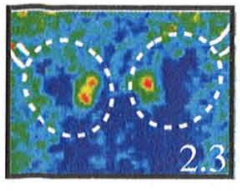

b

(i)

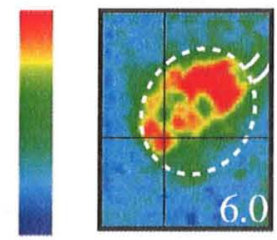

(ii)

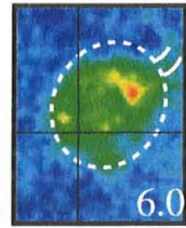

(iii)

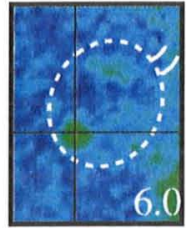

(iv)

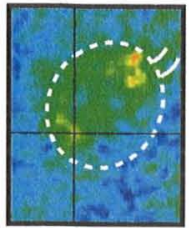

(ii)

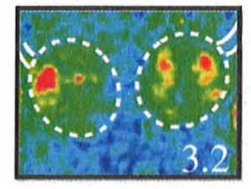

(v)

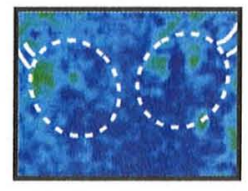

(iii)

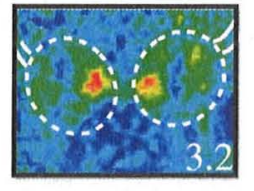

(vi)

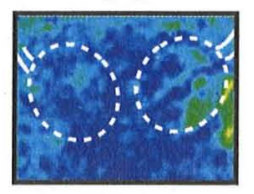

d

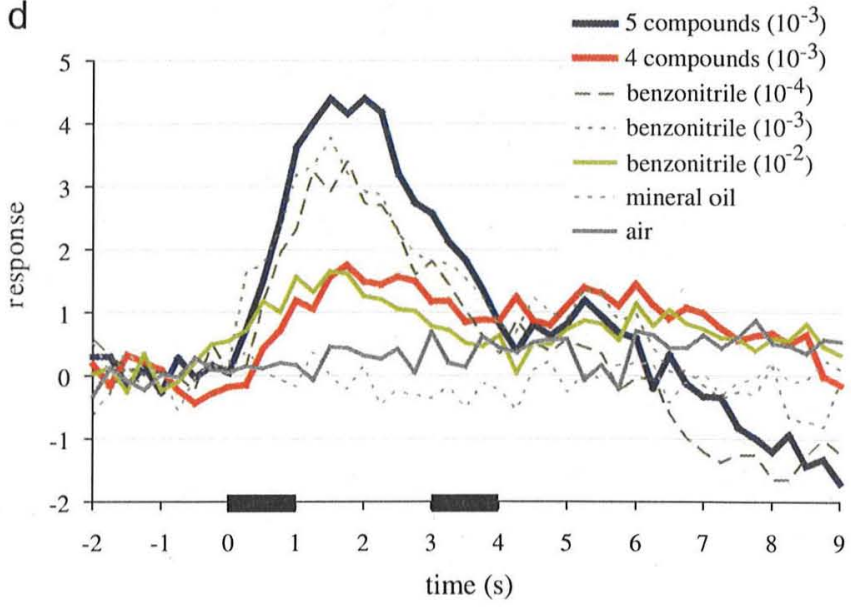

Fig. 2. (a) View of both antennal lobes $(A L)$ in a representative female moth stained with calcium green $A M$ (black bar: $100 \mu \mathrm{m})$; (b) response in the right $\mathrm{AL}$ of the same individual as in (a) during stimulation with (i) the benzonitrile-containing 5-compound mixture (at 10 ${ }^{-2}$ ); (ii) the 4-compound mixture (at $10^{-2}$ ); (iii) benzonitrile (at $10^{-4}$ ). Note the focal response to benzonitrile in a single glomerulus (glomerulus A) (hairline-cross). Shown in (iv) is the arithmetical subtraction of the glomerular response to the 4-compound mixture from the response to the 5-compound mixture; without mixture synergism this calculation should correspond to the response to benzonitrile (at $10^{-4}$ ) as shown in (iii). Note that the pattern involves more glomeruli and higher response strengths; (c) stimulation with either (i) ( \pm ) linalool (at $10^{-2}$ ), (ii) ( $Z$ )-3-hexen-1-yl acetate, (iii) benzaldehyde, or (iv) benzonitrile (each at $10^{-3}$ ), as well as with (v) mineral oil and (vi) blank; (d) for glomerulus A, time traces of calcium signals to five odorants and two controls. (b-d) show relative changes in fluorescence $(\Delta F / F)$, in false-color coding (see scale in (b)). Minimum was 0 in all images, and maxima for false-color scales are shown inside each picture.

by each of the three different mixtures was a combination of the signals recorded for the single odors (Figs. 2b and c), indicating no (or very weak) mixture interactions at the level of odor representation in the AL.

The 5-compound mixture elicited strong activation of one additional glomerulus (glomerulus A) (Fig. 2b-i) leading to a different pattern of activated glomeruli in comparison with that evoked by either the 3-compound mixture (data not shown) or the 4-compound mixture (Fig. 2b-ii). As indicated above, glomerulus A was identified based on its characteristically strong response to benzonitrile when offered alone at low concentrations (Fig. 2b-iii). Remarkably, signals recorded in this glomerulus were significantly stronger when benzonitrile was included in a mixture than when offered alone despite of the 4.5 times smaller amount of benzonitrile present in the mixture $(t$-test; df $=12 ; t$-value $=6.46, P<0.001 ; n=8$ and 6$)$. This result is also illustrated in Fig. 2b (i-iv) with a representative female moth using false-color coding pictures. An arithmetical subtraction of the pattern of glomerular responses to the 4-compound mixture (at $10^{-2}$ )
(Fig. 2b-ii) from the corresponding response to the 5-compound mixture (at $10^{-2}$ ) (Fig. 2b-i) revealed strong signals remaining in glomerulus $\mathrm{A}$ (shown in Fig. 2b-iv). Calcium signals remaining after this subtraction were stronger than the response recorded in this glomerulus to benzonitrile alone at $10^{-4}$, despite the lesser amount of benzonitrile contained in the mixture. These results indicate a synergistic effect originating from one or more of the additional compounds present in the 5-compound mixture, an interaction that was investigated in more detail (see physiological experiment 2).

Mixtures elicited spatially distributed patterns of activated glomeruli. The three green leaf volatiles (i.e., $[Z]-3-$ hexen-1-ol, [E]-2-hexenal, and [Z]-3-hexen-1-yl acetate) and the two aromatics (benzaldehyde and benzonitrile) activated distinct areas of the AL of the female moths (Fig. 2c). Glomeruli located in the dorso-lateral area of the AL were broadly tuned and responded to most odorants when tested at the lowest concentration and to all of them when offered at high concentration. In contrast, glomeruli located in the medial-ventral section of the AL showed 

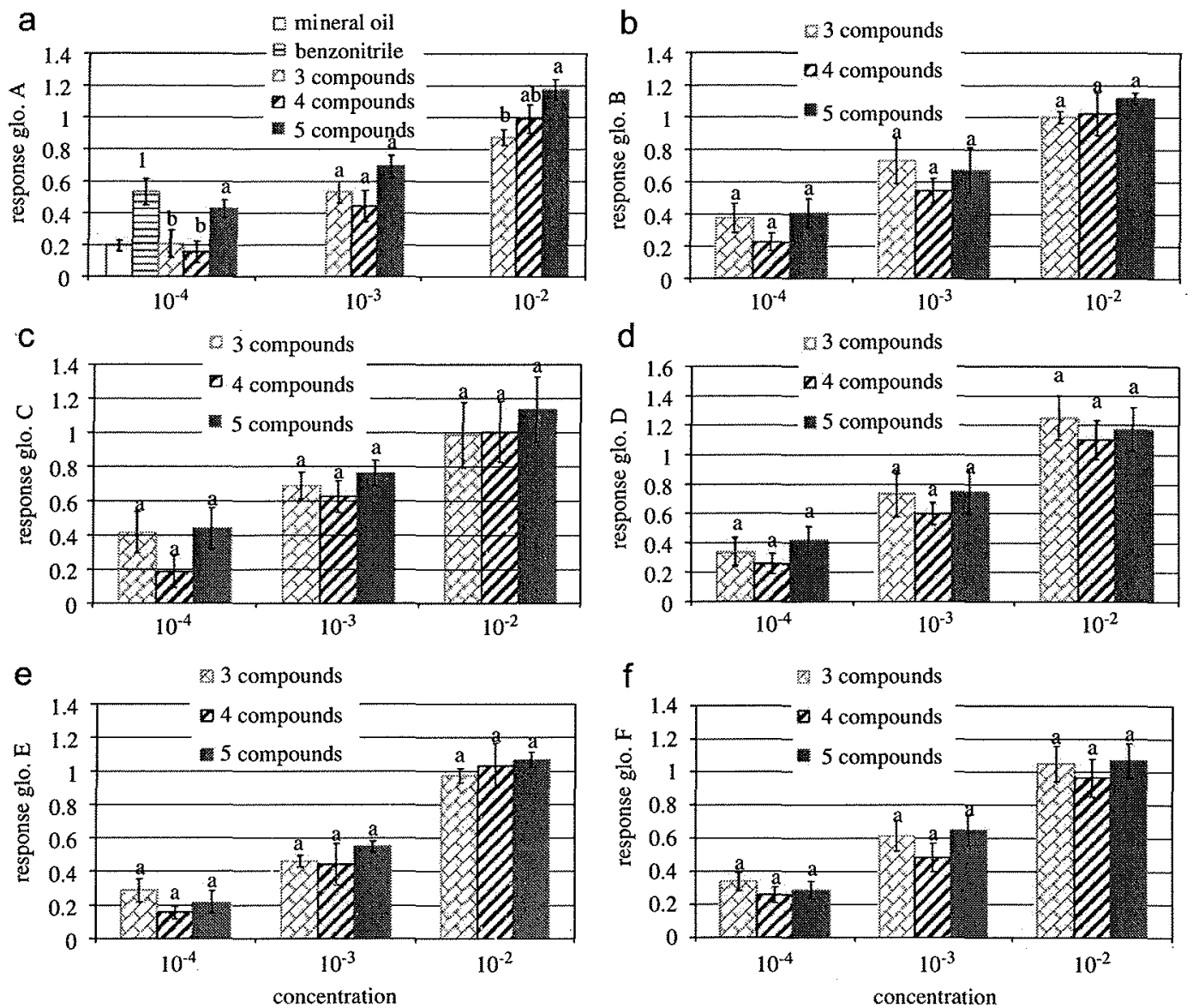

Fig. 3. Dose-response relationships of calcium signals recorded in response to three different odor mixtures recorded in each of the six different glomeruli identified (glo. A-F). In glomerulus A the responses to mineral oil (solvent) and to benzonitrile (at $10^{-4}$ ) are shown for comparison. 1: The absolute amount of benzonitrile at $10^{-4}$ is $\sim 100.4 \mathrm{ng} / \mu \mathrm{L}$, but because benzonitrile constitutes only $0.15 \%$ of the 5 -compound mixture, this amount is $\sim 456$ times greater than the amount of benzonitrile present in the 5-compound mixture at the same concentration $\left(10^{-4}\right)(\sim 0.22 \mathrm{ng} / \mu \mathrm{L})$. Values represent averaged responses ( \pm SEM) (normalized data, $n=8$ ). Within each concentration, bars superscribed by the same letter are not significantly different based on Tukey HSD tests at $P=0.05$.

narrower tuning and their responses were more specific to benzaldehyde and benzonitrile. Almost negligible calcium signals were induced by the controls mineral oil (solvent) and blank (i.e., the carrier nitrogen).

The time course of calcium signals recorded were typified as fast upstroke pattern reaching the maximum intensity within $1 \mathrm{~s}$ after stimulus onset, as shown in Fig. $2 \mathrm{~d}$ for two mixtures (at $10^{-3}$ ) and benzonitrile (at three concentrations) for glomerulus $\mathrm{A}$. The second odor stimulation given $3 \mathrm{~s}$ after the first one elicited small delayed responses in most cases (Fig. 2d) and were not further investigated in this study.

\subsection{Dose-response relationships of synthetic mixtures and individual odors}

Each of the odors tested (alone or in mixtures) fell within the dynamic range of stimulus-response relationship of this olfactory system. As shown in Figs. 3a-f for the three mixtures evaluated in the six glomeruli identified, the dose-response relationships were monotonic. Monotonic relationships between odor concentration and response strength were also noted for the individual odors evaluated except for glomerulus A when benzonitrile was tested alone (Fig. 4). In some of the female moths tested this glomerulus was already saturated when stimulated with benzonitrile at the intermediate concentration (i.e., at $10^{-3}$ ) and at the highest concentration tested the response in glomerulus $\mathrm{A}$ was greatly reduced. At the same time, an adjacent glomerulus that responded most strongly to benzaldehyde responded to high benzonitrile concentrations (data not shown).

The concentration series data also revealed that calcium signals recorded in glomerulus $A$ were significantly stronger in response to the 5-compound mixture (at $10^{-4}$ ) compared to the other two mixtures (ANOVA $F_{2,14}=4.2$; $P=0.036$ ). This was the only glomerulus identified in which the presence of benzonitrile in a mixture 

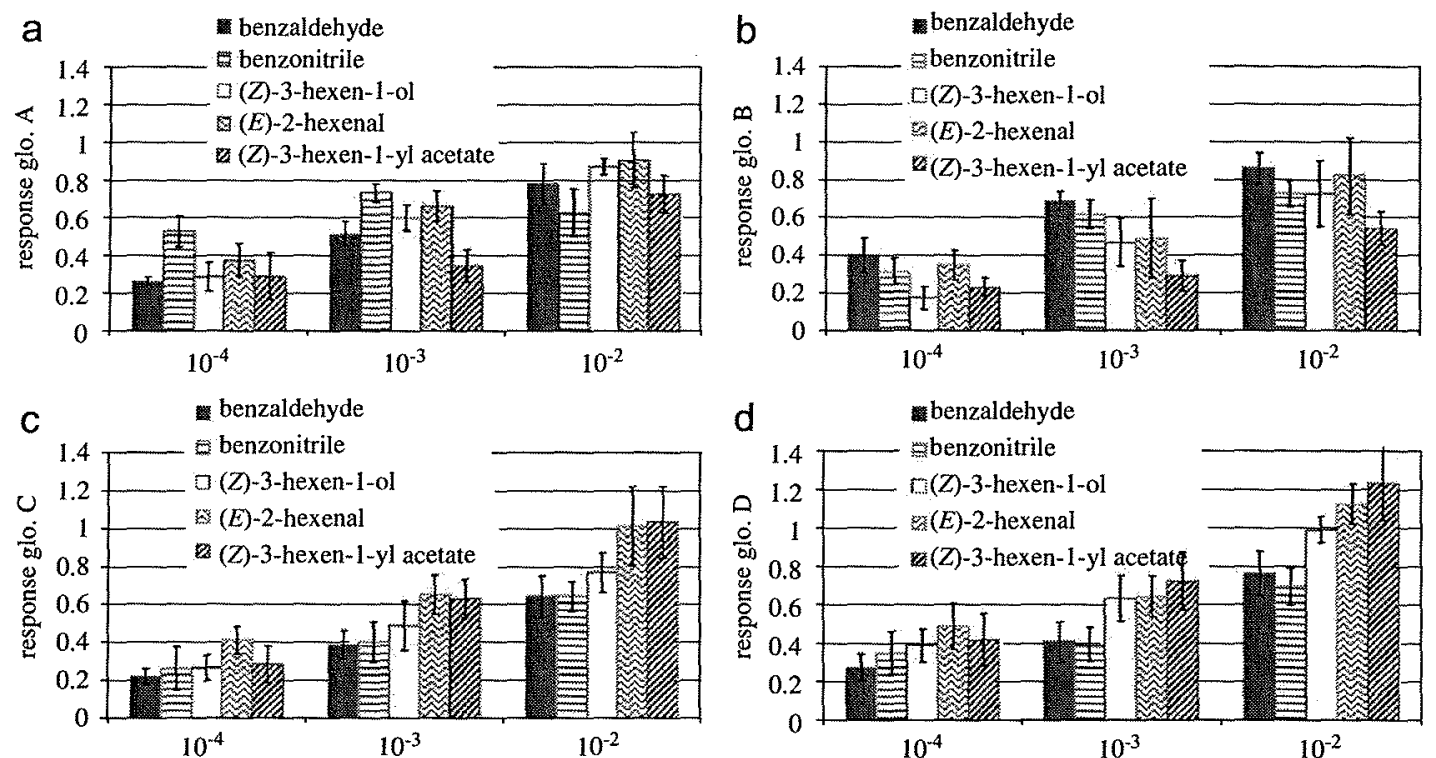

benzaldehyde
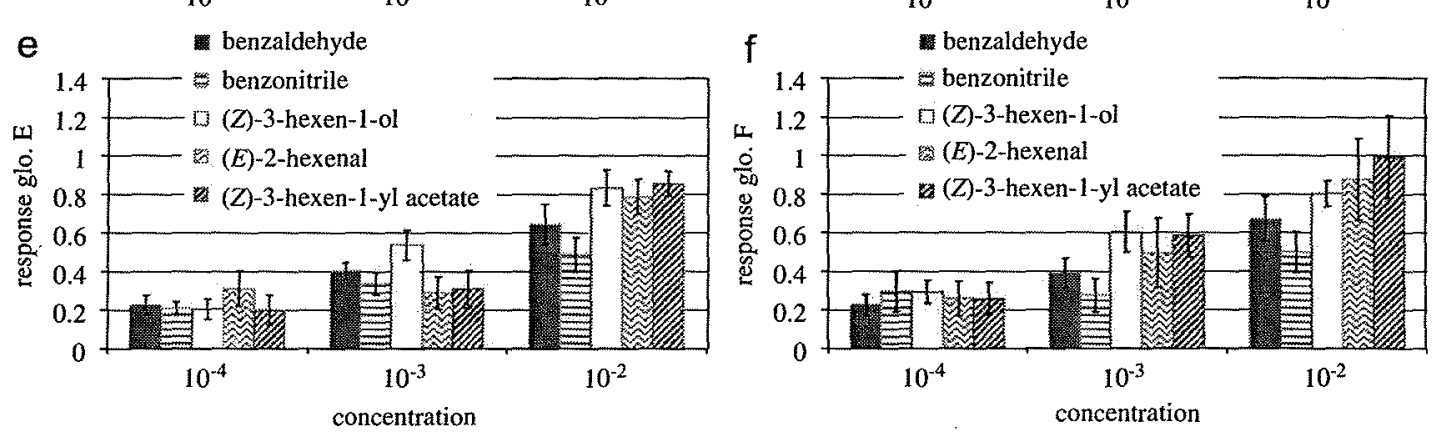

Fig. 4. Dose-response relationships of signals recorded in response to five individual compounds in each of the six different glomeruli identified (glo. A $-F$ ). Values represent averaged responses $( \pm \mathrm{SEM})$ (normalized data, $n=8$ ).

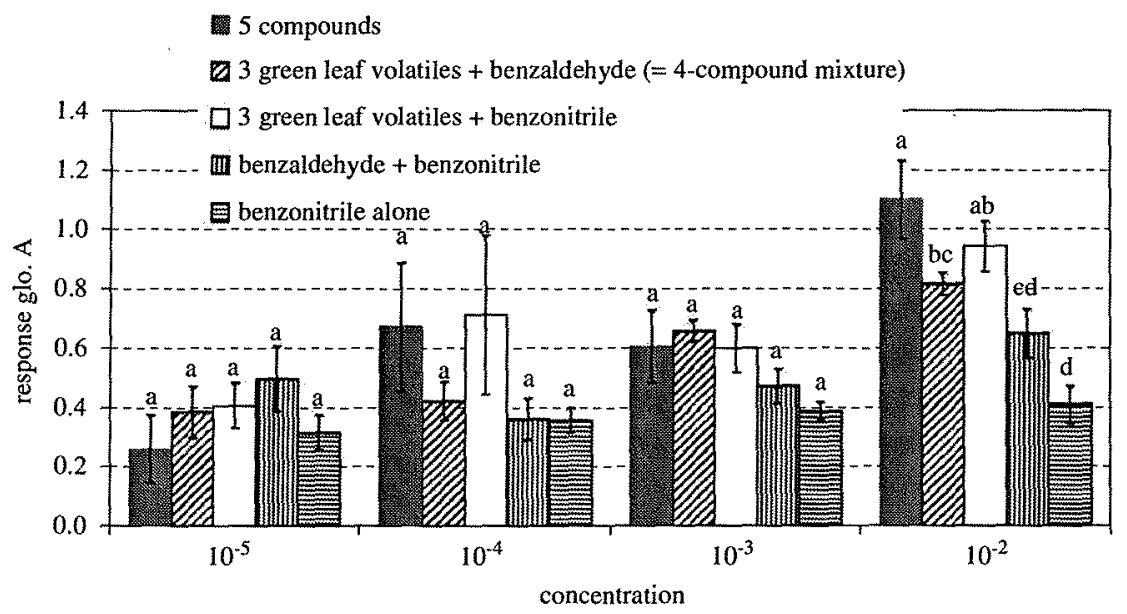

Fig. 5. For each of four different concentrations $\left(10^{-5}-10^{-2}\right)$, averaged responses $( \pm \mathrm{SEM})$ (normalized data, $\left.n=4\right)$ to five odor treatments recorded in the mixture-sensitive glomerulus A (glo. A). Within each concentration, bars superscribed by the same letter are not significantly different based on Tukey HSD tests at $P=0.05$.

greatly enhanced the magnitude of its response. In fact, stimulation of antennae with either the 3-compound mixture or the 4-compound mixtures at low concentrations elicited a level of response on this glomerulus that was comparable to stimulation with the solvent (mineral oil) (Fig. 3a). 


\subsection{Odor-evoked activity in the mixture-sensitive glomerulus}

The synergistic effect noted in the first physiological experiment was confirmed here for the highest concentration of odorants tested (i.e., $10^{-2}$ ) (ANOVA; $F_{4,15}=9.8$; $P<0.001 ; n=4)$. At this concentration, the 5-compound mixture elicited significantly stronger responses in this glomerulus than any other mixture except for a mixture (not evaluated in our first experiment) that contained benzonitrile in association with the three green leaf volatiles and excluded benzaldehyde. Further, signals recorded in response to the 4-compound mixture evaluated before (i.e., the 3-compound mixture in combination with [E]-2-hexenal) were not different from the signals recorded in response to the binary combination of benzaldehyde and benzonitrile. Benzonitrile alone elicited the weakest responses (Fig. 5). For the remaining concentrations, no significant differences in calcium signals recorded were determined among the five treatments evaluated (ANOVA; $F_{4,15}=1.0 ; \quad P=0.45 ; \quad F_{4,15}=1.2 ; \quad P=0.36 ; \quad$ and $F_{4,15}=2.3 ; P=0.11$ for odorants at $10^{-5}, 10^{-4}$, and $10^{-3}$, respectively) (Fig. 5).

\section{Discussion}

Few compounds commonly dominate the composition of complex blends emitted by plants (Hern and Dorn, 2003). Consequently, one frequent approach towards elucidation of behaviorally effective compounds has focused on the quantitatively prevailing compounds or fractions (e.g., Natale et al., 2003). The role that minor compounds play in behavioral discrimination has received comparatively little attention. Some of these minor constituents of odor blends have shown to be important for adequate odor recognition (Masson and Mustaparta, 1990; Piñero and Dorn, 2007). However, the correct identification and behavioral evaluation of such compounds present in very small amounts have represented an enormous challenge for researchers. Here we document that trace amounts of benzonitrile synergized the behavioral response of mated female oriental fruit moths to a synthetic mixture of four major plant-derived volatiles. While benzonitrile per se is behaviorally ineffective (Piñero and Dorn, 2007), it elicits a significant attraction of the moths when included in a mixture that also contains three general green leaf volatiles and benzaldehyde at the natural ratio found in the headspace of the host plant. Remarkably, moths did not discriminate between this bioactive 5compound mixture and the natural blend of volatiles emitted from the host plant.

In our physiological experiments, calcium responses were successfully measured in the $\mathrm{AL}$ of female oriental fruit moth using the bath-applied calcium-dye staining technique. The patterns of glomerular input activity evoked by the different mixtures were given by the combination of the patterns of the components as shown previously in bees (Joerges et al., 1997; Deisig et al., 2006) and in the zebrafish (Tabor et al., 2004), indicating no (or very weak) interactions among the components of the mixtures.

The first physiological experiment revealed that the particular spatial pattern of activated glomeruli in response to the behaviorally active 5-compound mixture differed substantially from the pattern elicited by the behaviorally ineffective 3- and 4-compound mixtures. Remarkably, calcium signals that remained after the arithmetical subtraction of the pattern of glomerular responses to the 4-compound mixture (at $10^{-2}$ ) from the corresponding response to the 5-compound mixture (at $10^{-2}$ ) were stronger than the response recorded in glomerulus $A$ to benzonitrile alone at $10^{-4}$, despite the lesser amount of benzonitrile contained in the mixture. For instance, the absolute amount of benzonitrile at $10^{-4}$ was $\sim 100.4 \mathrm{ng} / \mu \mathrm{L}$, whereas the absolute amount of benzonitrile contained in the 5-compound mixture at $10^{-2}$ was $\sim 22.1 \mathrm{ng} / \mu \mathrm{L}$. This finding can only be explained by a synergistic effect triggered by the presence of minute amounts of benzonitrile in the 5-compound mixture. Benzonitrile represents only $0.15 \%$ of the 5 -compound mixture and this small amount of benzonitrile corresponds to its relative proportion in the natural blend. Our combined data hence indicate that the high-quality signals that are required for behavioral discrimination, which are given by the particular spatial pattern of glomeruli activated by the bioactive 5-compound mixture, are already reflected at the level of odor representation in the $\mathrm{AL}$ of the oriental fruit moth.

Generalist-type and specialist-type receptors are involved in the coding of plant odors (Bargmann, 2006). Recent examples of olfactory systems reported to possess both types of receptors include the moths Manduca sexta (L.) (Shields and Hildebrand, 2001) and $H$. virescens (Røstelien et al., 2005) as well as the tephritid fly Rhagolet is pomonella (Walsh) (Olsson et al., 2006). Our physiological data revealed the existence of a new type of glomerulus not reported before that shows specific synergisms to particular complex mixtures. This glomerulus is highly sensitive to minute amounts of benzonitrile, suggesting that the corresponding receptor is similarly sensitive. Whether the observed synergistic interaction occurs within the neural network of the AL, or might already occur at the level of olfactory sensilla or receptor cells on the antennae remains to be elucidated. Of the six glomeruli identified in the first physiological experiment, only in glomerulus $\mathrm{A}$ was the level of activation strongly dependent upon the number of compounds present in the mixture and reached its greatest magnitude when benzonitrile was incorporated into a mixture (see Figs. 3a and 5).

The second physiological experiment confirmed that the behaviorally effective 5-compound mixture was able to stimulate glomerulus $\mathrm{A}$ in a way that exceeded dramatically the level of activation induced by benzonitrile alone offered at the same concentration. This synergistic effect elicited by benzonitrile seems to play a crucial role in the 
final performance of the olfactory system of this moth as evidenced by attractant effect of the 5-compound mixture. As shown in our first physiological experiment, glomerulus A showed a remarkable ability to integrate the different chemical inputs contained in the most complex mixture evaluated involving five compounds. This finding is supported by data from the second physiological experiment which revealed a gradual increase in the strength of the response in this glomerulus with increases in the number of compounds in the mixture, a result that was not noted in any of the remaining five glomeruli identified.

It is known that for non-pheromonal systems key information about odor quality is generally transmitted to the brain by an across-fiber pattern which requires a complex evaluation of neural activities in the whole array of olfactory receptors, whereas labeled lines involve specialist receptors (Visser and de Jong, 1988). Our results are suggestive that the olfactory system of the oriental fruit moth may be a combination of across-fiber patterning with an important contribution of glomerulus A. However, the synergistic nature of this glomerulus shows that its behavioral role cannot be considered a "labeled-line" system, where its activity alone would signal the presence of a suitable substrate. Rather, the synergism that we document offers physiological evidence for a complex pattern extractor for behaviorally relevant mixtures already at the level of the first brain area involved. More research is needed in this direction.

In conclusion, our behavioral and physiological results provide strong evidence that minor components of a complex mixture can have a significant effect at the levels of behavior and odor representation at the peripheral olfactory stage and may even be determinant in the final perception of odor quality. Our combined findings indicate that the synergistic effect elicited by benzonitrile seems to be central, when present in a mixture that contains other key constituents, for adequate coding (at the physiological level) and discrimination (at the behavioral level) of odor mixtures that might be encountered in the dynamic and fluctuating environment of the natural habitat of this moth species.

\section{Acknowledgments}

We thank Christine Dittrich for outstanding technical assistance, and Paul Szyszka and Ana Silbering (University of Konstanz) for helpful discussions and support during optical imaging and associated data analysis. We also thank Silke Hein (ETH Zurich) and two anonymous reviewers for useful comments on the manuscript, as well as Marco Torriani and Johanna Häckermann for providing adult moths.

\section{References}

Bargmann, C.I., 2006. Comparative chemosensation from receptors to ecology. Nature 444, 295-301.
Bertschy, C., Turlings, T.C.J., Bellotti, A., Dorn, S., 1997. Chemically mediated attraction of three parasitoid species to mealybug-infested cassava leaves. Florida Entomologist 80, 383-395.

Bonferroni, C.E., 1936. Teoria statistica delle classi e calcolo delle probabilità. Pubblicazioni del $R$ Istituto Superiore di Scienze Economiche e Commerciali di Firenze 8, 3-62.

Bruce, T.J.A., 2005. Insect host location: a volatile situation. Trends in Plant Science 10, 269-274.

Carlsson, M.A., Hansson, B.S., 2003. Plasticity and coding mechanisms in the insect antennal lobe. In: Blomquist, G.J., Vogt, R.V. (Eds.), Insect Pheromone Biochemistry and Molecular Biology. Elsevier Academic Press, San Diego, CA, USA, pp. 699-728.

Carlsson, M.A., Chong, K.Y., Daniels, W., Hansson, B.S., Pearce, T.C., 2007. Component information is preserved in glomerular responses to binary odour mixtures in the moth Spodoptera littoralis. Chemical Senses 32, 433-443.

Christensen, T.A., Mustaparta, Hildebrand, J.G., 1991. Chemical communication in heliothine moths. Journal of Comparative Physiology A $169,259-274$.

Deisig, N., Giurfa, M., Lachnit, H., Sandoz, J.C., 2006. Neural representation of olfactory mixtures in the honeybee antennal lobe. European Journal of Neuroscience 24, 1161-1174.

Eigenbrode, S.D., Bernays, E.A., 1997. Evaluation of factors affecting host plant selection, with an emphasis on studying behaviour. In: Dent, D.R., Walton, M.P. (Eds.), Methods in Ecological and Agricultural Entomology. CAB International, Cambridge, pp. 147-169.

Galizia, C.G., Menzel, R., 2001. The role of glomeruli in the neural representation of odours: results from optical recording studies. Journal of Insect Physiology 47, 115-130.

Galizia, C.G., Vetter, R.S., 2005. Optical methods for analyzing odourevoked activity in the insect brain. In: Christensen, T.A. (Ed.), Methods in Insect Sensory Neuroscience. CRC Press, Boca Raton, Florida, USA, pp. 349-392.

Galizia, C.G., Nagler, K., Holldobler, B., Menzel, R., 1998. Odour coding is bilaterally symmetrical in the antennal lobes of honeybees (Apis mellifera). European Journal of Neuroscience 10, 2964-2974.

Galizia, C.G., Sachse, S., Mustaparta, H., 2000. Calcium responses to pheromones and plant odours in the antennal lobe of the male and female moth Heliothis virescens. Journal of Comparative Physiology 186, 1049-1063.

Hern, A., Dorn, S., 2003. Monitoring seasonal variation in apple fruit volatile emissions in situ using solid-phase microextraction. Phytochemical Analysis 14, 232-240.

Hern, A., Dorn, S., 2004. A female-specific attractant for the codling moth, Cydia pomonella, from apple fruit volatiles. Naturwissenschaften $91,77-80$.

Hughes, J., Hern, A., Dorn, S., 2004. Preimaginal environment influences adult flight in Cydia molesta (Lepidoptera:Tortricidae). Environmental Entomology 33, 1155-1162.

Joerges, J., Kuttner, A., Galizia, C.G., Menzel, R., 1997. Representations of odours and odour mixtures visualized in the honeybee brain. Nature $387,285-288$.

Lledo, P.M., Gheusi, G., Vincent, J.D., 2005. Information processing in the mammalian olfactory system. Physiological Reviews 85, 281-317.

Masson, C., Mustaparta, H., 1990. Chemical information-processing in the olfactory system of insects. Physiological Reviews 70, 199-245.

Mayer, M.S., Doolittle, R.E., 1995. Synergism of an insect sex pheromone specialist neuron: implications for component identification and receptor interactions. Journal of Chemical Ecology 21, 1875-1891.

Natale, D., Mattiacci, L., Hern, A., Pasqualini, E., Dorn, S., 2003. Response of female Cydia molesta (Lepidoptera:Tortricidae) to plant derived volatiles. Bulletin of Entomological Research 93, 335-342.

Nikonov, A.A., Leal, W.S., 2002. Peripheral coding of sex pheromone and behavioral antagonist in the Japanese beetle. Journal of Chemical Ecology 28, 1079-1093.

Olsson, S.B., Linn Jr., C.E., Roelofs, W.L., 2006. The chemosensory basis for behavioral divergence involved in sympatric host shifts: I. 
Characterizing olfactory receptor neuron classes responding to key host volatiles. Journal of Comparative Physiology A 192, 279-288.

Piñero, J.C., Dorn, S., 2007. Synergism between aromatic compounds and green leaf volatiles derived from the host plant underlies female attraction in the oriental fruit moth. Entomologia Experimentalis et Applicata 125, 185-194.

Røstelien, T., Stranden, M., Borg-Karlson, A.K., Mustaparta, H., 2005. Olfactory receptor neurons in two heliothine moth species responding selectively to aliphatic green leaf volatiles, aromatic compounds, monoterpenes and sesquiterpenes of plant origin. Chemical Senses 30, 443-461.

Rothschild, G.H.L., Vickers, R.A., 1991. Biology, ecology and control of the oriental fruit moth. In: van der Geest, L.P.S., Evenhuis, H.H. (Eds.), World Crop Pests, Tortricid Pests their Biology, Natural Enemies and Control, vol. 5. Elsevier Academic Publishers, The Netherlands, pp. 389-412.

Sachse, S., Rappert, A., Galizia, C.G., 1999. The spatial representation of chemical structures in the antennal lobe of honeybees: steps towards the olfactory code. European Journal of Neuroscience 11, 3970-3982. Schoonhoven, L.M., Jermy, T., van Loon, J.J.A., 1998. Insect-Plant Biology: From Physiology to Evolution. Chapman \& Hall, London, UK.

Shields, V.D.C., Hildebrand, J.G., 2001. Recent advances in insect olfaction, specifically regarding the morphology and sensory physiology of antennal sensilla of the female sphinx moth Manduca sexta. Microscopy Research and Technology 55, 307-329.
Skiri, H.T., Galizia, C.G., Mustaparta, H., 2004. Representation of primary plant odorants in the antennal lobe of the moth Heliothis virescens using calcium imaging. Chemical Senses 29, 253-267.

Smith, B.H., Getz, W.M., 1994. Nonpheromonal olfactory processing in insects. Annual Review of Entomology 39, 351-375.

StatSoft Inc., 2001. STATISTICA (data analysis software system), version 6. 〈www.statsoft.com〉.

Tabor, R., Yaksi, E., Weislogel, J.M., Friedrich, R.W., 2004. Processing of odour mixtures in the zebrafish olfactory bulb. Journal of Neuroscience 24, 6611-6620.

Tasin, M., Bäckman, A.C., Coracini, M., Casado, D., Ioriatti, C., Witzgall, P., 2007. Synergism and redundancy in a plant volatile blend attracting grapevine moth females. Phytochemistry 68, 203-209.

Vallat, A., Dorn, S., 2005. Changes in volatile emissions from apple trees and associated response of adult female codling moths over the fruitgrowing season. Journal of Agriculture and Food Chemistry 53, 4083-4090.

Vallat, A., Gu, H., Dorn, S., 2005. How rainfall, relative humidity and temperature influence volatile emissions from apple trees in situ. Phytochemistry 66, 1540-1550.

Visser, J.H., 1986. Host odour perception in phytophagous insects. Annual Review of Entomology 31, 121-144.

Visser, J.H., de Jong, R., 1988. Olfactory coding in the perception of semiochemicals. Journal of Chemical Ecology 14, 2005-2018. 